\title{
Quantum interference shifts in laser spectroscopy with elliptical polarization
}

\author{
Pedro Amaro, ${ }^{1, *}$ Filippo Fratini, ${ }^{2}$ Laleh Safari, ${ }^{3}$ Aldo Antognini, ${ }^{4,5}$ Paul Indelicato, ${ }^{6}$ Randolf Pohl, ${ }^{7}$ and José Paulo Santos ${ }^{1}$ \\ ${ }^{1}$ Laboratório de Instrumentação, Engenharia Biomédica e Física da Radiação (LIBPhys-UNL), Departamento de Física, \\ Faculdade de Cêincias e Tecnologia, FCT, Universidade Nova de Lisboa, 2829-516 Caparica, Portugal \\ ${ }^{2}$ Atominstitut, TU Wien, 1020 Vienna, Austria \\ ${ }^{3}$ IST Austria, Am Campus 1, A-3400 Klosterneuburg, Austria \\ ${ }^{4}$ Institute for Particle Physics, ETH Zurich, 8093 Zurich, Switzerland \\ ${ }^{5}$ Paul Scherrer Institute, 5232 Villigen-PSI, Switzerland \\ ${ }^{6}$ Laboratoire Kastler Brossel, Sorbonne Université, Université Pierre et Marie Curie-Paris 06, École Normale Supérieure, \\ Collège de France, CNRS, Case 74, 4 place Jussieu, F-75005 Paris, France \\ ${ }^{7}$ Max Planck Institute of Quantum Optics, 85748 Garching, Germany \\ (Received 5 November 2015; published 31 December 2015)
}

\begin{abstract}
We investigate the quantum interference shifts between energetically close states, where the state structure is observed by laser spectroscopy. We report a compact and analytical expression that models the quantum interference induced shift for any admixture of circular polarization of the incident laser and angle of observation. An experimental scenario free of quantum interference can thus be predicted with this formula. Although this study is exemplified here for muonic deuterium, it can be applied to any other laser spectroscopy measurement of $n s-n^{\prime} p$ frequencies of a nonrelativistic atomic system, via an $n s \rightarrow n^{\prime} p \rightarrow n^{\prime \prime} s$ scheme.
\end{abstract}

DOI: 10.1103/PhysRevA.92.062506

PACS number(s): 32.70.Jz, 36.10.Ee, 32.10.Fn, 32.80.Wr

\section{INTRODUCTION}

As was pointed by Low [1], a spectral line profile can only be described by a conventional Lorentzian profile up to certain limit of accuracy. Beyond this limit, known as the resonant approximation, the full quantum interference (QI) between the main resonant channels and other nonresonant channels makes the spectral lines asymmetric. Consequently, if Lorentzian functions are employed to fit the distorted profiles, a mismatch between the obtained centroid frequency and the actual line frequency occurs [2-5]. These QI-induced shifts are specific for a particular measurement, and its quantification is mandatory for all high-precision spectroscopy experiments aimed at a resolution beyond the resonant approximation. A known example is the case of the $1 s-2 s$ transition in hydrogen, which prompted many QI theoretical studies applied to various experimental methods, namely continuous-wave photon scattering [3,6], two-photon excitation [4,7], and direct two-photon frequency-comb spectroscopy [8]. Other atomic systems that have been considered include the helium fine structure and lithium hyperfine structure, where it was shown that the negligence of the QI effects is the cause of many discrepant measurements [5,9-16]. On the other hand, a recent analysis noted that QI shifts can be safely neglected in the cesium hyperfine structure [17]. Recently, the geometric and polarization properties of the QI shifts were investigated in laser spectroscopy, both experimentally and theoretically $[5,16]$, and it was found that the QI shifts vanish for a particular angle of linear polarization, the so-called "magic angle." This result was applied recently to minimize QI shifts in laser spectroscopy of hydrogen [18].

The aim of this article is twofold: First, we continue and conclude the investigation of the QI shifts in laser spectroscopy of muonic atoms [19]. We confirm that the conclusions of [19], namely that the line centers are not affected on a relevant level

\footnotetext{
*pdamaro@fct.unl.pt
}

by QI effects in muonic atoms, hold true even for a hypothetical admixture of circular polarized light. Second, we extend the theoretical description developed for linear polarized photons in Ref. [5] to elliptically polarized photons. A compact and analytical formula that models the QI shifts for any angle of observation, angle, and degree of circular polarization is presented here that may be beneficial to forthcoming laser experiments.

\section{THEORY}

Laser spectroscopy is often modeled by the physical process of resonant photon scattering $[5,18,19]$. Here, we quantify the QI effects involved in the precise determination of the $n s-2 p$ transition frequencies by exciting the $n s \rightarrow 2 p$ transition and detecting the $2 p \rightarrow 1 s$ fluorescence decay. The overall process to be considered is thus $n s \rightarrow 2 p \rightarrow 1 s$ photon scattering. Following the second-order theory of KramersHeisenberg [20], the differential scattering cross section of photon scattering from an initial $2 s_{J_{i}}^{F_{i}}$ state to a final $1 s_{J_{f}}^{F_{f}}$ state is given in atomic units by

$$
\frac{d \sigma}{d \Omega}\left(\hat{\boldsymbol{\varepsilon}}_{1}, \theta\right)=\frac{1}{\left(2 F_{i}+1\right)} \sum_{\substack{m_{i}, F_{f}, m_{f}, J_{f} \\ \hat{\varepsilon}_{2}}}\left|\mathcal{M}_{i \rightarrow f}^{\hat{\varepsilon}_{1}, \hat{\varepsilon}_{2}}\right|^{2},
$$

where $F_{i}$ and $F_{f}$ are the initial and final total angular momenta, and $m_{i}$ and $m_{f}$ are the respective projections along the quantization axis. The second-order amplitude $\mathcal{M}_{i \rightarrow f}^{\hat{\varepsilon}_{1}, \hat{\varepsilon}_{2}}$ involves a summation over the entire atomic spectrum [21], which in the near-resonant region comprises only the $v \equiv$ $2 p_{J_{v}}^{F_{v}}$ intermediate states. In the dipole and rotating-wave approximation, it is given by

$$
\mathcal{M}_{i \rightarrow f}^{\hat{\varepsilon}_{1} \hat{\boldsymbol{\varepsilon}}_{2}}=\sum_{F_{v}, m_{v}, J_{v}} \frac{\left\langle f\left|\alpha \boldsymbol{p} \cdot \hat{\boldsymbol{\varepsilon}}_{2}\right| v\right\rangle\left\langle v\left|\alpha \boldsymbol{p} \cdot \hat{\boldsymbol{\varepsilon}}_{1}\right| i\right\rangle}{\omega_{v i}-\omega_{1}-i \Gamma_{v} / 2},
$$

with $\omega_{v i}$ being the $2 s_{J_{i}}^{F_{i}}-2 p_{J_{v}}^{F_{v}}$ transition frequencies between $|v\rangle$ and $|i\rangle, \Gamma_{\nu}$ is the $2 p_{J}^{F}$ linewidth that is assumed to be 
independent of the hyperfine state $\Gamma_{2 p_{J}^{F}} \equiv \Gamma_{2 p}, \boldsymbol{p}$ is the linear momentum, $\hat{\boldsymbol{\varepsilon}}_{1}$ and $\hat{\boldsymbol{\varepsilon}}_{2}$ are the polarization vectors of the incoming and scattered photon, respectively (see below), and $\alpha$ is the fine-structure constant. Energy conservation sets $\omega_{2}-\omega_{1}=$ $\omega_{i}-\omega_{f}$ between the incident $\left(\omega_{1}\right)$ and scattered $\left(\omega_{2}\right)$ frequencies and initial $\left(\omega_{i}\right)$ and final $\left(\omega_{f}\right)$ atomic state frequencies.

The angular distribution of the scattered photon is given by the polar angle $\theta$, included in the scattering plane defined by both photon momenta $\left(\boldsymbol{k}_{1}\right.$ and $\left.\boldsymbol{k}_{2}\right)$, as illustrated in Fig. 1. We consider the experimental scenario of the second photon's polarization $\hat{\boldsymbol{\varepsilon}}_{2}$ not being detected, which is often the case in laser spectroscopy experiments [5,22,23]. Following the procedure of Istomin et al. [24], we parametrize the incident elliptical polarized photons as $\hat{\boldsymbol{\varepsilon}}_{1}=\left(\hat{\boldsymbol{\varepsilon}}_{l}+i \eta \hat{\boldsymbol{\varepsilon}}_{l}^{\perp}\right) / \sqrt{1+\eta^{2}}$. As shown in Fig. 1, $\hat{\boldsymbol{\varepsilon}}_{l}$ is defined with an angle $\chi$ relative to the scattering plane, and $\hat{\boldsymbol{\varepsilon}}_{l}^{\perp}=-\hat{\boldsymbol{k}}_{1} \times \hat{\boldsymbol{\varepsilon}}_{l}$. The circular admixture is often quantified by using the degree of circular polarization $P_{c}$ that is defined by the difference between left and right spherical amplitudes $\left(\left|\varepsilon_{1}^{1}\right|^{2}\right.$ and $\left.\left|\varepsilon_{1}^{-1}\right|^{2}\right)$ of the incident polarization, normalized to the total amplitude $\left(\left|\hat{\boldsymbol{\varepsilon}}_{1}\right|^{2}=1\right)$. By using the previous parametrization of $\hat{\boldsymbol{\varepsilon}}_{1}$, it is related with the admixture parameter $(-1 \leqslant \eta \leqslant 1)$ by

$$
P_{c}=\left|\varepsilon_{1}^{1}\right|^{2}-\left|\varepsilon_{1}^{-1}\right|^{2}=\frac{2 \eta}{1+\eta^{2}}
$$

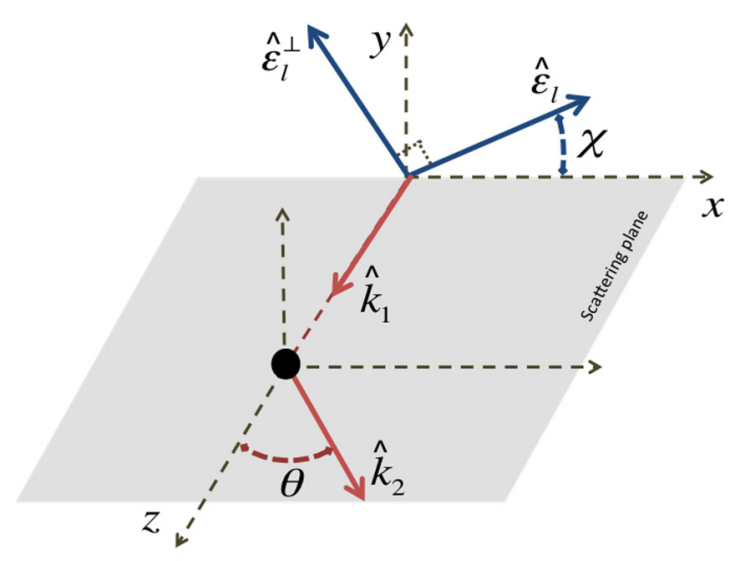

FIG. 1. (Color online) Geometry of the photon scattering for incident elliptical polarized photons with momentum $\boldsymbol{k}_{1}$ and polarization $\hat{\boldsymbol{\varepsilon}}_{1}=\left(\hat{\boldsymbol{\varepsilon}}_{l}+i \eta \hat{\boldsymbol{\varepsilon}}_{l}^{\perp}\right) / \sqrt{1+\eta^{2}}$, and scattered photon momentum $\boldsymbol{k}_{2}$, uniquely defined by $\theta . \hat{\boldsymbol{\varepsilon}}_{l}$ is tilted by $\chi$ relative to the scattering plane and $\hat{\boldsymbol{\varepsilon}}_{l}^{\perp}$ is orthogonal to $\hat{\boldsymbol{\varepsilon}}_{l}$. Both vectors are defined in the $y x$ plane. The second photon's polarization is not observed and is not illustrated.

By using standard angular algebra [25], Eq. (1) can be further rearranged in a suitable form to study the QI shifts in terms of Lorentzian terms $\Lambda_{J_{i} J_{v}}^{F_{i} F_{v}}(\theta, \chi, \eta)$ and cross-terms $\Xi_{J_{i} J_{v} J_{v^{\prime}}}^{F_{i} F_{v} F_{v^{\prime}}}(\theta, \chi, \eta)[5,19]$. The result is given by

$$
\frac{d \sigma}{d \Omega}(\theta, \chi, \eta)=\frac{\omega_{1} \omega_{2}^{3} \mathcal{S}_{f v i}^{2}}{\left(2 F_{i}+1\right)}\left(\sum_{F_{v}, J_{v}} \frac{\Lambda_{J_{i} J_{v}}^{F_{i} F_{v}}(\theta, \chi, \eta)}{\left(\omega_{\nu i}-\omega_{1}\right)^{2}+\left(\Gamma_{\nu} / 2\right)^{2}}+\sum_{\left(F_{v}^{\prime}, J_{\nu}^{\prime}\right)>\left(F_{v}, J_{\nu}\right)} \frac{\Xi_{J_{i} J_{v} J_{v^{\prime}}}^{F_{i} F_{v} F_{v^{\prime}}(\theta, \chi, \eta)}}{\left(\omega_{\nu i}-\omega_{1}-i \Gamma_{\nu} / 2\right)\left(\omega_{\nu^{\prime} i}-\omega_{1}+i \Gamma_{\nu} / 2\right)}\right)
$$

with the quantities defined by

$$
\Lambda_{J_{i} J_{v}}^{F_{i} F_{v}}(\theta, \chi, \eta)=\sum_{m_{i}, F_{f}, m_{f}, J_{f}, \hat{\boldsymbol{\varepsilon}}_{2}}\left|\Omega_{J_{i} J_{v} J_{f}}^{F_{J} F_{v} F_{f}}\left(\hat{\boldsymbol{\varepsilon}}_{1}, \hat{\boldsymbol{\varepsilon}}_{2}\right)\right|^{2} \text {, and } \Xi_{J_{i} J_{v} J_{v}^{\prime}}^{F_{i} F_{v} F_{v}^{\prime}}(\theta, \chi, \eta)=2 \operatorname{Re}\left[\sum_{\substack{m_{i}, F_{f} \\ m_{f}, J_{f}, \hat{\boldsymbol{\varepsilon}}_{2}}} \Omega_{J_{i} J_{v} J_{f}}^{F_{i} F_{v} F_{f}}\left(\hat{\boldsymbol{\varepsilon}}_{1}, \hat{\boldsymbol{\varepsilon}}_{2}\right)\left(\Omega_{J_{i} J_{v}^{\prime} J_{f}}^{F_{F} F^{\prime} F_{f}}\left(\hat{\boldsymbol{\varepsilon}}_{1}, \hat{\boldsymbol{\varepsilon}}_{2}\right)\right)^{*}\right],
$$

having all the geometrical and polarization dependencies in terms of

$$
\Omega_{J_{i} J_{v} J_{f}}^{F_{i} F_{v} F_{f}}\left(\hat{\boldsymbol{\varepsilon}}_{1}, \hat{\boldsymbol{\varepsilon}}_{2}\right)=\left[J_{v} F_{\nu}\right] \sqrt{\left[F_{f}, F_{i}, J_{f}, J_{i}\right]}\left(\begin{array}{ccc}
J_{f} & 1 & J_{v} \\
1 / 2 & 0 & -1 / 2
\end{array}\right)\left(\begin{array}{ccc}
J_{v} & 1 & J_{i} \\
1 / 2 & 0 & -1 / 2
\end{array}\right)\left\{\begin{array}{ccc}
F_{f} & 1 & F_{v} \\
J_{v} & I & J_{f}
\end{array}\right\}\left\{\begin{array}{ccc}
F_{v} & 1 & F_{i} \\
J_{i} & I & J_{v}
\end{array}\right\} \Theta_{F_{f}}^{F_{v}} F_{i} \cdot
$$

Here, $I$ is the nuclear spin, $\mathcal{S}_{f v i}$ contains all radial integrals, and

$$
\Theta_{F_{f} F_{i}}^{F_{v}}=\sum_{\lambda_{1}, \lambda_{2}} \sum_{m_{v}}(-1)^{\lambda_{1}+\lambda_{2}+m_{v}+m_{f}+1} \varepsilon_{1}^{\lambda_{1}} \varepsilon_{2}^{\lambda_{2} *}\left(\begin{array}{ccc}
F_{f} & 1 & F_{v} \\
-m_{f} & \lambda_{2} & m_{v}
\end{array}\right)\left(\begin{array}{ccc}
F_{v} & 1 & F_{i} \\
-m_{v} & \lambda_{1} & m_{i}
\end{array}\right) .
$$

The cross-terms $\Xi(\theta, \chi, \eta)$ (angular momentum quantities are omitted for brevity) contain all interference between neighboring resonances, and if they are zero, then Eq. (4) is reduced to a sum of independent Lorentzian components. As demonstrated by Brown et al. [5] for the case of linear incident polarized photons $\left(\eta=P_{c}=0\right)$, both theoretically and experimentally, these cross-terms can be parametrized as $\Xi(\theta, \chi, 0)=b_{2} P_{2}(\sin \theta \cos \chi)$ (angles defined in our geometry [19]), where $P_{2}(x)=\left(3 x^{2}-1\right) / 2$ is the second-order Legendre polynomial. The coefficient $b_{2}$ depends on the angular momenta of the states participating in the transition. Therefore, there are particular combinations of $\theta^{\prime}$ and $\chi^{\prime}$, where QI effects vanish, that can be obtained by solving $\Xi\left(\theta^{\prime}, \chi^{\prime}, 0\right)=0$. For the case of $\theta^{\prime}=90^{\circ}$, the angle $\chi^{\prime}=\operatorname{arcos}(1 / \sqrt{3}) \approx 54.7^{\circ}$ is referred to as a "magic angle" in the literature [5].

\section{RESULTS AND DISCUSSION}

To investigate the role of elliptical polarization on the $\delta_{\mathrm{QI}}$ shifts, and as a continuation of a previous investigation [19], we choose the resonance with the largest induced shift in muonic deuterium, which is the resonance $2 s_{1 / 2}^{F=3 / 2} \rightarrow$ $2 p_{3 / 2}^{F=1 / 2}$. Following the procedure in Ref. [19], we evaluate the $\delta_{\mathrm{QI}}$ by fitting a simulated spectrum of Eq. (4), which would 
(a) $\chi=0^{\circ}$

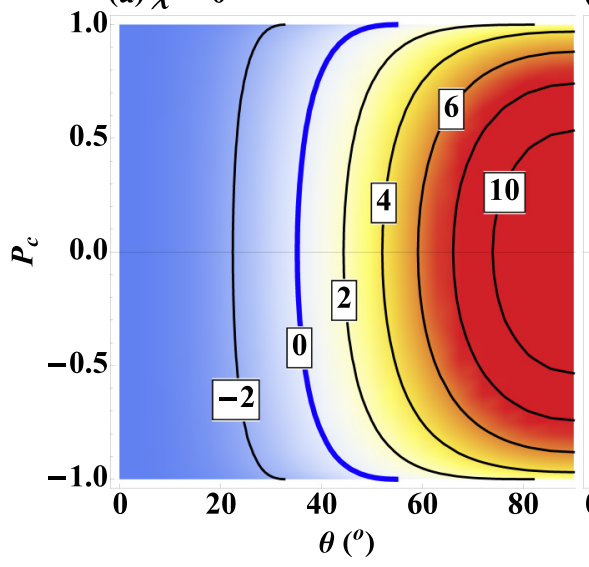

(b) $\theta=90^{\circ}$

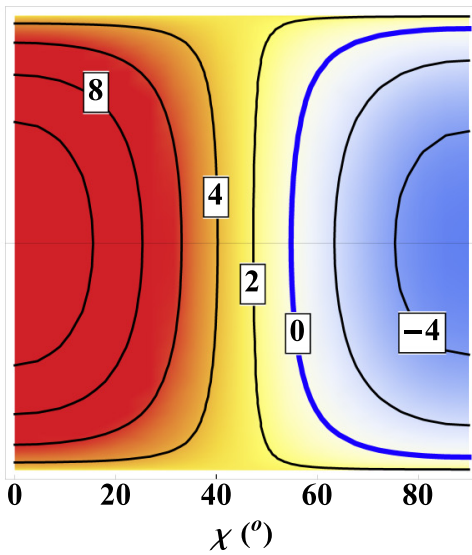

(c) $\chi=90^{\circ}$

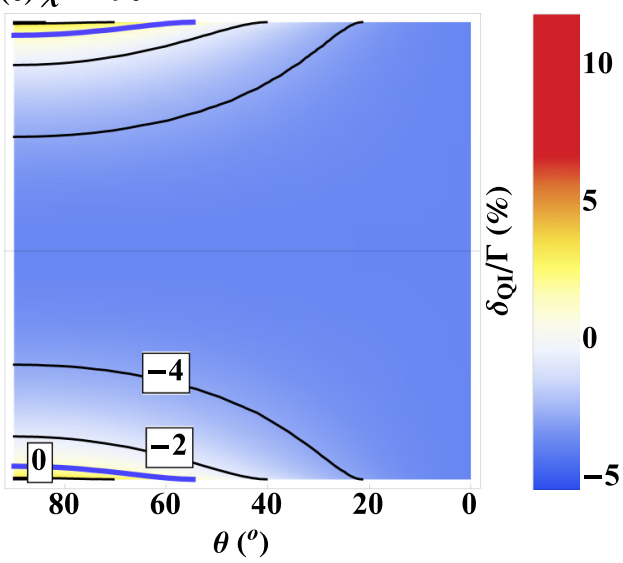

FIG. 2. (Color online) Contour plot of the QI shift normalized to the linewidth $\delta_{\mathrm{QI}} / \Gamma(\%)$ for the $2 s_{1 / 2}^{F=3 / 2} \rightarrow 2 p_{3 / 2}^{F=1 / 2}$ resonance of muonic deuterium as a function of the degree of circular polarization $P_{c}$ for the cases of (a) $\chi=0^{\circ}$, (b) $\theta=90^{\circ}$, and (c) $\chi=90^{\circ}$. The thicker blue lines show the parameter space where the QI shifts vanish.

be observed by a pointlike detector with a sum of Lorentzian profiles. Figure 2 displays the computed $\delta_{\mathrm{QI}}$ in units of the linewidth for all values of $P_{c}$, and for three cases of $\chi=0^{\circ}$ (a), $\theta=90^{\circ}(\mathrm{b})$, and $\chi=90^{\circ}$ (c).

As can be seen in Fig. 2(b), $\delta_{\mathrm{QI}}$ is proportional to $P_{2}(\cos \chi)$ for linear polarized photons $\left(\eta=P_{c}=0\right)$, as mentioned in Ref. [5]. Consequently, $\delta_{\mathrm{QI}}=0$ for the angle of polarization $\chi^{\prime} \approx 54.7^{\circ}$. Additionally, the points at $\chi=0^{\circ}\left(\delta_{\mathrm{QI}}^{\|}\right)$and $\chi=$ $90^{\circ}\left(\delta_{\mathrm{QI}}^{\perp}\right)$ with $P_{c}=0$ represent the QI shifts listed in Ref. [19] $\left(\delta_{\mathrm{QI}}^{\|}=12.3 \%\right.$ and $\left.\delta_{\mathrm{QI}}^{\perp}=-4.9 \%\right)$.

As can be observed in Fig. 2(a), for $P_{c}=0$ and $\chi=0^{\circ}$, there is an additional "magic angle" of observation $\theta^{\prime} \approx 35.3^{\circ}$ where $\delta_{\mathrm{QI}}$ vanishes. On the other hand, for the case of $P_{c}=0$ and $\chi=90^{\circ}$ represented in Fig. 2(c), $\delta_{\mathrm{QI}}$ is independent of $\theta$. This is expected since the dipole pattern of the differential cross section depends only on the angle between polarization and scattered direction, which for $\chi=90^{\circ}$ is independent of $\theta$.

Moreover, Fig. 2(b) shows that the contribution of the circular admixture to $\delta_{\mathrm{QI}}$ is bounded by the values of $\delta_{\mathrm{QI}}^{\|}$ and $\delta_{\mathrm{QI}}^{\perp}$ at $P_{c}=0$. Thus, any possible or uncontrolled circular admixture reduces the QI contribution relative to the linear case and a pointlike detector.

For circular polarized photons $\left(\eta=P_{c}= \pm 1\right), \delta_{\mathrm{QI}}$ is independent of $\chi$ [see Fig. 2(b)] since the differential cross section depends only on $\chi$ through the $x$ projection of $\boldsymbol{\varepsilon}_{1}$ in the scattering plane, given by $\left(\cos ^{2} \chi+\eta^{2} \sin \chi\right) /\left(1+\eta^{2}\right)$, which for $\eta= \pm 1$ is constant. The value of $\delta_{\mathrm{QI}} / \Gamma \approx 2.8 \%$ in this setting is the same as that at $\chi=45^{\circ}$ and any $\eta$ or $P_{c}$, following the same reasoning.

The symmetry between helicities $\left[\delta_{\mathrm{QI}}\left(\theta, \chi, P_{c}\right)=\delta_{\mathrm{QI}}\right.$ $\left.\left(\theta, \chi,-P_{c}\right)\right]$, displayed in Fig. 2 , is a consequence of not considering the scattered polarization and the final magnetic sublevel structure in the measurement scheme.

The laser system employed by the Charge Radius Experiment with Muonic Atoms (CREMA) collaboration was designed for linear polarization [26,27], but some small admixture of $10 \%$ of circular polarization cannot be excluded. Thus, it is worthwhile to evaluate the QI shift with this circular admixture for the CREMA geometry setup $\delta_{\mathrm{QI}}^{*}$, following steps similar to those performed in Ref. [19]. The obtained value of $\delta_{\mathrm{OI}}^{*} / \Gamma=0.3 \%$ for $P_{c}= \pm 0.1$ can be compared with the value of $\delta_{\mathrm{QI}}^{*} / \Gamma=0.13 \%$ [19] for $P_{c}=0$ (linear polarization). This shift of $0.3 \%$ of the linewidth sets a maximum threshold of $\delta_{\mathrm{QI}}^{*}$ for all resonances of the muonic atoms considered. Thus, even in the remote case of the laser having a small amount of circular polarization, QI shifts can be neglected for the present experimental resolution of muonic transitions that have been measured to date [22,23].

The scattering process considered here is of dipolar type, which is characterized by an angular dependency of the form $\frac{d \sigma}{d \Omega} \sim a\left(\chi, P_{c}\right)+b\left(\chi, P_{c}\right) \cos ^{2} \theta$ [20]. Following the formula of $P_{2}$, this dipole angular distribution can always be rewritten as $a^{\prime}\left(\chi, P_{c}\right)+b^{\prime}\left(\chi, P_{c}\right) P_{2}(\sin \theta)$. Thus, the cross-terms $\Xi(\theta, \chi, \eta)$ can also be expressed as $c\left(\chi, P_{c}\right)+d\left(\chi, P_{c}\right) P_{2}(\sin \theta)$. The analytical forms of $c\left(\chi, P_{c}\right)$ and $d\left(\chi, P_{c}\right)$, obtained after evaluation of Eqs. (5)-(7), can be further rearranged in order to include the $\chi$ and $P_{c}$ dependencies in $P_{2}$. This is accomplished with the help of $P_{2}(a \sqrt{b})=b P_{2}(a)+(b-1) / 2$ and with Eq. (3). We found, after this procedure, that the cross-terms $\Xi(\theta, \chi, \eta)$ have a compact and analytical expression for the angular and polarization properties, which is given by

$$
\Xi\left(\theta, \chi, P_{c}\right)=b_{2} P_{2}\left(\sin \theta \sqrt{\frac{\cos (2 \chi) \sqrt{1-P_{c}^{2}}+1}{2}}\right) .
$$

The coefficient $b_{2}$ contains the information on the angular quantum numbers involved in a particular transition. The respective values for many resonances in muonic atoms are listed in Ref. [19]. Equation (8) models the angular and polarization dependency of $\Xi\left(\theta, \chi, P_{c}\right)$ for any transition $n s \rightarrow n^{\prime} p \rightarrow n^{\prime \prime} s$ in an atomic system, under the premise of nonrelativistic and dipole approximation frameworks. We can thus use Eq. (8) to predict regions of the "magic values" $\left(\theta^{\prime}, \chi^{\prime}, P_{c}^{\prime}\right)$, where $\delta_{\mathrm{QI}}=0$, by solving $\Xi\left(\theta^{\prime}, \chi^{\prime}, P_{c}^{\prime}\right)$ equal to zero. This can be used to design accordingly a spectroscopy experiment insensitive to line-pulling effects. For example, the blue contour with $\delta_{\mathrm{QI}}=0$ in Fig. 2(b), which was computed numerically, is 
approximately equal to $\chi^{\prime}=\operatorname{arcos}\left[-1 /\left(3 \sqrt{1-P_{c}^{2}}\right)\right] / 2$, which for $\chi^{\prime}=90^{\circ}$ gives $P_{c}^{\prime} \approx \pm 0.94$. For circular polarization $P_{c}= \pm 1$, a quick inspection of Eq. (8) shows that $\delta_{\mathrm{QI}}=0$ occurs for the angle of observation $\theta^{\prime}=54.7^{\circ}$, as is also shown in Figs. 2(a) and 2(c).

Apart from immediate application in laser spectroscopy of atomic systems, Eq. (8) might also be applied to molecular physics and chemistry, where line mixing occurs due to interference of neighborhood molecular states. Without further observation of the internal structure of the target, the dipole pattern of photon scattering is quite general and independent of the target being an atom or a molecule [28]. Essentially, the angular and polarization dependency of interference shifts, included in Eq. (8), might be extended to molecular techniques based on photon scattering, such as resonant x-ray emission spectroscopy (XES) [29,30], Raman spectroscopy [31,32], and laser spectroscopy [33-35], where interference effects or line mixing might play a significant role.

\section{SUMMARY}

In summary, we investigated the contribution of an admixture of circular polarization to the QI shift by considering incident elliptical-polarized photons. Calculations performed for the CREMA detector setup revealed a negligible impact of QI effects for the maximum expected admixture of circular polarization.

We presented a compact and analytical expression that models the dependency of the angular and polarization properties to the QI shift. As a generalization of a similar expression for linear polarization [5], this one contains a degree of circular polarization. Although we considered here a particular resonance of muonic deuterium, as an illustrative example this expression can be applied to any transition $n s \rightarrow n^{\prime} p \rightarrow n^{\prime \prime} s$ in any nonrelativistic atomic system. Thus, this equation can be used to design a spectroscopy apparatus to measure $n s-n^{\prime} p$ frequencies in a scheme free of quantum interference shifts by optimizing the detector geometry, the laser polarization, and the laser direction.

\section{ACKNOWLEDGMENTS}

This research was supported in part by Fundação para a Ciência e a Tecnologia (FCT), Portugal, through Projects No. PEstOE/FIS/UI0303/2011 and No. PTDC/FIS/117606/2010, financed by the European Community Fund FEDER through the COMPETE. P.A. acknowledges the support of the FCT under Contract No. SFRH/BPD/92329/2013. R.P. acknowledges the support from the European Research Council (ERC) through StG. No. 279765. F.F. acknowledges support by the Austrian Science Fund (FWF) through the START Grant Y 591-N16. L.S. acknowledges financial support from the People Programme (Marie Curie Actions) of the European Union's Seventh Framework Programme (FP7/2007-2013) under REA Grant Agreement No. [291734]. A.A. acknowledges the support of the Swiss National Science Foundation Projects No. 200021L 138175 and No. 200020 159755. Laboratoire Kastler Brossel is "UMR n ${ }^{\circ} 8552$ " of the École Normale Supérieure, CNRS, Collége de France and Sorbonne University - Universiy Pierre and Marie Curie-Paris 06. P.I. is a member of the Helmholtz Alliance HA216/EMMI.
[1] F. Low, Phys. Rev. 88, 53 (1952).

[2] L. Labzowsky, V. Karasiev, and I. Goidenko, J. Phys. B 27, L439 (1994).

[3] L. N. Labzowsky, D. A. Solovyev, G. Plunien, and G. Soff, Phys. Rev. Lett. 87, 143003 (2001).

[4] L. Labzowsky, G. Schedrin, D. Solovyev, E. Chernovskaya, G. Plunien, and S. Karshenboim, Phys. Rev. A 79, 052506 (2009).

[5] R. C. Brown, S. Wu, J. V. Porto, C. J. Sansonetti, C. E. Simien, S. M. Brewer, J. N. Tan, and J. D. Gillaspy, Phys. Rev. A 87, 032504 (2013).

[6] U. D. Jentschura and P. J. Mohr, Can. J. Phys. 80, 633 (2002).

[7] L. N. Labzowsky, G. Schedrin, D. Solovyev, and G. Plunien, Can. J. Phys. 85, 585 (2007).

[8] D. C. Yost, A. Matveev, E. Peters, A. Beyer, T. W. Hänsch, and T. Udem, Phys. Rev. A 90, 012512 (2014).

[9] M. Horbatsch and E. A. Hessels, Phys. Rev. A 82, 052519 (2010).

[10] A. Marsman, M. Horbatsch, and E. A. Hessels, Phys. Rev. A 86, 012510 (2012).

[11] A. Marsman, M. Horbatsch, and E. A. Hessels, Phys. Rev. A 86, 040501 (2012).

[12] A. Marsman, E. A. Hessels, and M. Horbatsch, Phys. Rev. A 89, 043403 (2014).

[13] A. Marsman, M. Horbatsch, and E. A. Hessels, Phys. Rev. A 91, 062506 (2015).
[14] A. Marsman, M. Horbatsch, and E. A. Hessels, J. Phys. Chem. Ref. Data 44, 031207 (2015).

[15] G. P. Feng, X. Zheng, Y. R. Sun, and S. M. Hu, Phys. Rev. A 91, 030502 (2015).

[16] C. J. Sansonetti, C. E. Simien, J. D. Gillaspy, J. N. Tan, S. M. Brewer, R. C. Brown, S. Wu, and J. V. Porto, Phys. Rev. Lett. 107, 023001 (2011).

[17] G. W. Truong, J. D. Anstie, E. F. May, T. M. Stace, and A. N. Luiten, Nat. Commun. 6, 8345 (2015).

[18] A. Beyer, L. Maisenbacher, K. Khabarova, A. Matveev, R. Pohl, T. Udem, T. W. Hänsch, and N. Kolachevsky, Phys. Scr. 2015, 014030 (2015).

[19] P. Amaro, B. Franke, J. J. Krauth, M. Diepold, F. Fratini, L. Safari, J. Machado, A. Antognini, F. Kottmann, P. Indelicato, R. Pohl, and J. P. Santos, Phys. Rev. A 92, 022514 (2015).

[20] R. Loudon, The Quantum Theory of Light (Oxford Science, Oxford, 2000).

[21] L. Safari, P. Amaro, S. Fritzsche, J. P. Santos, S. Tashenov, and F. Fratini, Phys. Rev. A 86, 043405 (2012).

[22] R. Pohl, A. Antognini, F. Nez, F. D. Amaro, F. Biraben, J. M. R. Cardoso, D. S. Covita, A. Dax, S. Dhawan, L. M. P. Fernandes, A. Giesen, T. Graf, T. W. Hänsch, P. Indelicato, L. Julien, C.-Y. Kao, P. Knowles, E.-O. Le Bigot, Y.-W. Liu, J. A. M. Lopes, L. Ludhova, C. M. B. Monteiro, F. Mulhauser, T. Nebel, P. Rabinowitz, J. M. F. dos Santos, L. A. Schaller, K. Schuhmann, 
C. Schwob, D. Taqqu, J. F. C. A. Veloso, and F. Kottmann, Nature (London) 466, 213 (2010).

[23] A. Antognini, F. Nez, K. Schuhmann, F. D. Amaro, F. Biraben, J. M. R. Cardoso, D. S. Covita, A. Dax, S. Dhawan, M. Diepold, L. M. P. Fernandes, A. Giesen, A. L. Gouvea, T. Graf, T. W. Hänsch, P. Indelicato, L. Julien, C.-Y. Kao, P. Knowles, F. Kottmann, E.-O. Le Bigot, Y.-W. Liu, J. A. M. Lopes, L. Ludhova, C. M. B. Monteiro, F. Mulhauser, T. Nebel, P. Rabinowitz, J. M. F. dos Santos, L. A. Schaller, C. Schwob, D. Taqqu, J. F. C. A. Veloso, J. Vogelsang, and R. Pohl, Science 339, 417 (2013).

[24] A. Y. Istomin, E. A. Pronin, N. L. Manakov, S. I. Marmo, and A. F. Starace, Phys. Rev. Lett. 97, 123002 (2006).

[25] M. E. Rose, Elementary Theory of Angular Momentum (Wiley, New York, 1957).

[26] A. Antognini, F. D. Amaro, F. Biraben, J. M. R. Cardoso, C. A. N. Conde, D. S. Covita, A. Dax, S. Dhawan, L. M. P. Fernandes, and T. W. Hänsch, Opt. Commun. 253, 362 (2005).

[27] A. Antognini, K. Schuhmann, F. D. Amaro, F. Biraben, A. Dax, A. Giesen, T. Graf, T. W. Hänsch, P. Indelicato, L. Julien,
K. Cheng-Yang, P. E. Knowles, F. Kottmann, E. Le Bigot, L. Yi-Wei, L. Ludhova, N. Moschuring, F. Mulhauser, T. Nebel, F. Nez, P. Rabinowitz, C. Schwob, D. Taqqu, and R. Pohl, IEEE J. Quantum Electron. 45, 993 (2009).

[28] D. P. C. a. T. Thirunamachandran, Molecular Quantum Electrodynamics: An Introduction to Radiation-Molecule Interactions (Dover, Mineola, New York, 1984).

[29] Y. Luo, H. Ågren, and F. Gel'mukhanov, Phys. Rev. A 53, 1340 (1996).

[30] Y. Horikawa, T. Tokushima, A. Hiraya, and S. Shin, Phys. Chem. Chem. Phys. 12, 9165 (2010).

[31] X. Lu, S. Venugopalan, H. Kim, M. Grimsditch, S. Rodriguez, and A. K. Ramdas, Phys. Rev. B 83, 195126 (2011).

[32] J. G. Duque, H. Telg, H. Chen, A. K. Swan, A. P. Shreve, X. Tu, M. Zheng, and S. K. Doorn, Phys. Rev. Lett. 108, 117404 (2012).

[33] R. Berman, P. Duggan, P. M. Sinclair, A. D. May, and J. R. Drummond, J. Mol. Spectrosc. 182, 350 (1997).

[34] M. Mudrich, F. Stienkemeier, G. Droppelmann, P. Claas, and C. P. Schulz, Phys. Rev. Lett. 100, 023401 (2008).

[35] H. Goto, H. Katsuki, H. Ibrahim, H. Chiba, and K. Ohmori, Nat. Phys. 7, 383 (2011). 